\title{
Knowledge Modeling Framework for System Engineering Projects
}

\author{
Olfa Chourabi ${ }^{1}$, Yann Pollet ${ }^{2}$, and Mohamed Ben Ahmed ${ }^{3}$
}

\begin{abstract}
System Engineering (SE) projects encompass knowledge-intensive tasks that involve extensive problem solving and decision making activities among interdisciplinary teams. Management of knowledge emerging in previous SE projects is vital for organizational process improvement. To fully exploit this intellectual capital, it must be made explicit and shared among system engineers. In this context, we propose a knowledge modelling framework for system engineering projects. Our main objective is to provide a semantic description for knowledge items created and/or used in system engineering processes in order to facilitate their reuse. The framework is based on a set of layered ontologies where entities such as domain concepts, actors, decision processes, artefacts, are interlinked to capture explicit as well as implicit engineering project knowledge.
\end{abstract}

\section{Introduction}

System Engineering (SE) is an interdisciplinary approach to enable the realization of successful systems. It is defined as an iterative problem solving process aiming

\footnotetext{
${ }^{1}$ Olfa chourabi

CNAM, 292, rue saint martin, paris

RIADI, campus universitaire de la Manouba.

Olfa.chourabi@voila.fr

${ }^{2}$ Yann pollet

CNAM, 292, rue saint martin, paris.pollet@cnam.fr

${ }^{3}$ Mohamed Ben Ahmed

RIADI, campus universitaire de la Manouba, mohamed.benahmed@riadi.rnu.tn
} 
at transforming user's requirements into a solution satisfying the constraints of: functionality, cost, time and quality. [1]

System engineering projects involve the definition of multiple artifacts that present different formalization degrees, such as requirements specification, system architecture, and hardware/software components. Transitions between the project phases stem from decision making processes supported both by generally available domain and design knowledge.

We argue that Knowledge about engineering processes constitutes one of the most valuable assets for SE organizations. Most often, this knowledge is only known implicitly, relying heavily on the personal experience background of system engineers. To fully exploit this intellectual capital, it must be made explicit and shared among project teams. Consistent and comprehensive knowledge management methods need to be applied to capture and integrate the individual knowledge items emerging in the course of a system engineering project. Knowledge management (KM) is a scientific discipline that stems from management theory and concentrates on the systematic creation, leverage, sharing and reuse of knowledge resources in a company. [2] Knowledge management approaches are generally divided into personalization approaches that focus on human resources and communication, and codification approaches that emphasize the collection and organization of knowledge [3].

In this paper, we only consider the latter approach. Special focus is put on the comprehensive modeling of system engineering project knowledge. This knowledge partly resides in the product itself, while a lot of different types of knowledge are generated during the engineering processes. The background information such as why engineers came up with the final shape or geometry, what constraints were to be considered in engineering processes, and so on, can not be found either [4]. In other words, most of design rationale either disappear or exist partially in the form of engineering documents. In such setting, the most critical issue is related to the construction of a structured representation for engineering project knowledge modeling that record engineers' ideas and reasoning processes for a specific issue. This representation must be based on a formal language with expressive semantics, in order to perform computable operations on the recorded knowledge items and to improve their retrieval. Ontological engineering [5], which is the successor to knowledge engineering, has been expected to resolve the problem of semantic based knowledge modeling. In the engineering domain, the typical expectations for ontologies are: interoperability among engineering supporting systems, semantic constraints for modeling, implicit knowledge capture and knowledge systematization, [6]. In the context of our research, we use the term "ontology" as a formal structure providing a basis of knowledge systematization. [6] We propose a set of layered ontologies for representing the relevant engineering project entities and their Knowledge Modeling Framework for System Engineering Projects. 


\section{Background and motivation}

\subsection{System Engineering processes}

System engineering (SE) is an interdisciplinary approach to enable the realization of successful systems. It is defined as an iterative problem solving process aiming at transforming user's requirements into a solution satisfying the constraints of: functionality, cost, time and quality [1]. This process is usually comprised of the following seven tasks: State the problem, Investigate alternatives, Model the system, Integrate, Launch the system, Assess performance, and Re-evaluate. These functions can be summarized with the acronym SIMILAR: State, Investigate, Model, Integrate, Launch, Assess and Re-evaluate. [7]. This Systems Engineering Process is shown in Figure 1.

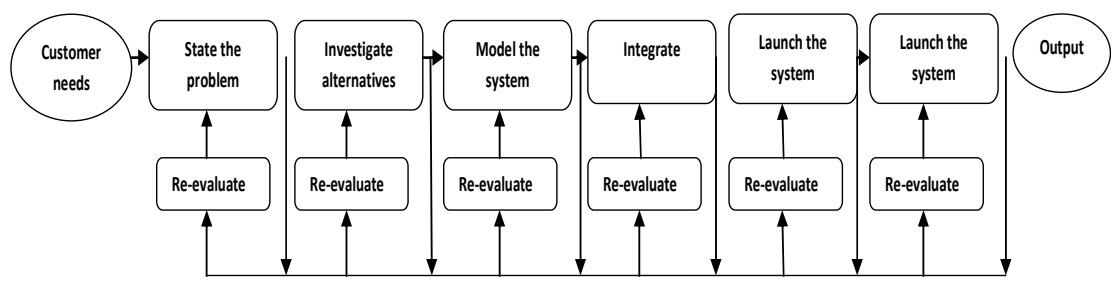

Fig 1: System Engineering Process [7]

It is important to note that the System Engineering Process is not sequential. Tasks are performed in a parallel and iterative manner. At each step a comprehensive set of possible engineering models arises witch are progressively combined and refined to define the target system.

Because of its inherent creative nature, it is a special case of business process. It is poorly structured and, as a rule, evolves in an unpredictable manner. In such highly dynamic settings with continuously changing requirements, the overwhelming majority of the engineering ways of working are not properly formalized, but are heavily based on the experience knowledge of the human performers.

As a consequence, engineering support environments have further to deal with the systematic collection of experience from previous project cycles and its dissemination and utilization from analogous problem solving contexts in the future. [8]. in section 3, we present a knowledge modeling framework that acts as a backend for what we expect to be a "Next generation of engineering support environment" i.e.: "knowledge centric" rather than "data centric" [12]. 


\subsection{Knowledge Management issues in SE}

The Above-delineated characteristics of SE processes show that a significant amount of knowledge is involved to solve a mix of ill- and well-defined problems. System engineers require topic knowledge (learned from text books and courses) and episodic knowledge (experience) [9].

One of the main problems in SE processes is the lack of capture and access to knowledge underpinning the design decisions and the processes leading to those decisions $[10,11]$.

System Engineers spend large portions of their time searching through vast amounts of corporate legacy data and catalogs searching for existing solutions which can be modified to solve new problems or to be assembled into a new device. This requires utilizing databases or online listings of text, images, and computer aided design (CAD) data. Browsing and navigating such collections are based on manually-constructed categorizations which are error prone, difficult to maintain, and often based on an insufficiently dense hierarchy. Search functionality is limited to inadequate keyword matching on overly simplistic attributes; it lacks the formal framework to support automated reasoning. [8]

In this paper, we focus on the knowledge modeling issue which is often considered as the first step in developing Knowledge-Based Systems (KBS). The aim of this process is to understand the types of data structures and relationships within which knowledge can be held, and reasoned with. We use ontologies to describe the knowledge model in a formal representation language with expressive semantics.

In order to determine the basic building blocks of the knowledge repository, we introduce the notion of "SE-Project Asset" as the smallest granularity in the system experience knowledge. "SE-Project Asset", represent an integrated structure that capture product and process knowledge in engineering situations in conformance to set of layered ontologies.

\section{Knowledge Modeling Framework for System Engineering Projects}

In this section, our framework for knowledge modeling in system engineering projects is described. It structures the traces of engineering in the form of semantic descriptions based on a system engineering ontology. Section 3.1 introduces the so-called "SE general Ontology", and Section 3.2 describes the modeling layers considered for semantic knowledge capture. 


\subsection{System Engineering General Ontology}

Basically, our model aims at specifying explicitly the facets describing an "SEProject Asset".

We choose to model these description facets with ontologies. In the knowledge engineering community, a definition by Gruber is widely accepted; that is, "explicit specification of conceptualization" [13], where conceptualization is "a set of objects which an observer thinks exist in the world of interest and relations between them" [14]. In engineering domain, ontology is considered "a system (systematic, operational and prescriptive definitions) of fundamental concepts and relationships which shows how a model author views the target world and which is shared in a community as building blocks for models. [6]

By instantiating these ontological concepts, concrete "SE-Project Asset" could be stored in a system engineering repository for future reuse. Furthermore, the ontology itself can serve as a communication base about the products and processes e.g. for exploring domain knowledge for system engineers.

We propose three description facets to capture the "SE-Project Asset". These three facets are arranged in a "SE general ontology" that introduces top-level concepts describing products and processes, as well as their interrelations and dependencies, independently from any particular engineering domain or application.

The main idea is to capture the engineering products, engineering processes, the design rationale, and the domain concepts in order to provide a comprehensive and computable description for projects knowledge. These descriptions facets are arranged around the "SE-Project Asset" as the central concept for SE project knowledge modeling.

-Domain facet: contains basic concepts and relations for describing the content of engineering assets on a high semantic level. It can be regarded as domain ontology for system engineering. In order to capture all engineering artifacts in a comprehensive manner, we propose to integrate in this facet a systematic description of: domain requirements, domain functions and behavior, domain architecture, and domain physical components. This decomposition constitutes typical system engineering modeling areas. Nevertheless, they could be extended or restricted in function of the engineering domain and the knowledge modeling scope. We work on aligning this domain facet with the reference device ontology described in [15]. Figure 3 presents a high level description of a typical domain facet.

-Product facet: contains concepts and relations representing artifact types as well as their information model. In SE domain, a system is described with several views such as: contextual, dynamic, static, functional or organic. By formally 
relating modeling elements to domain concepts we could provide a systematic and semantic description of an engineering solution.

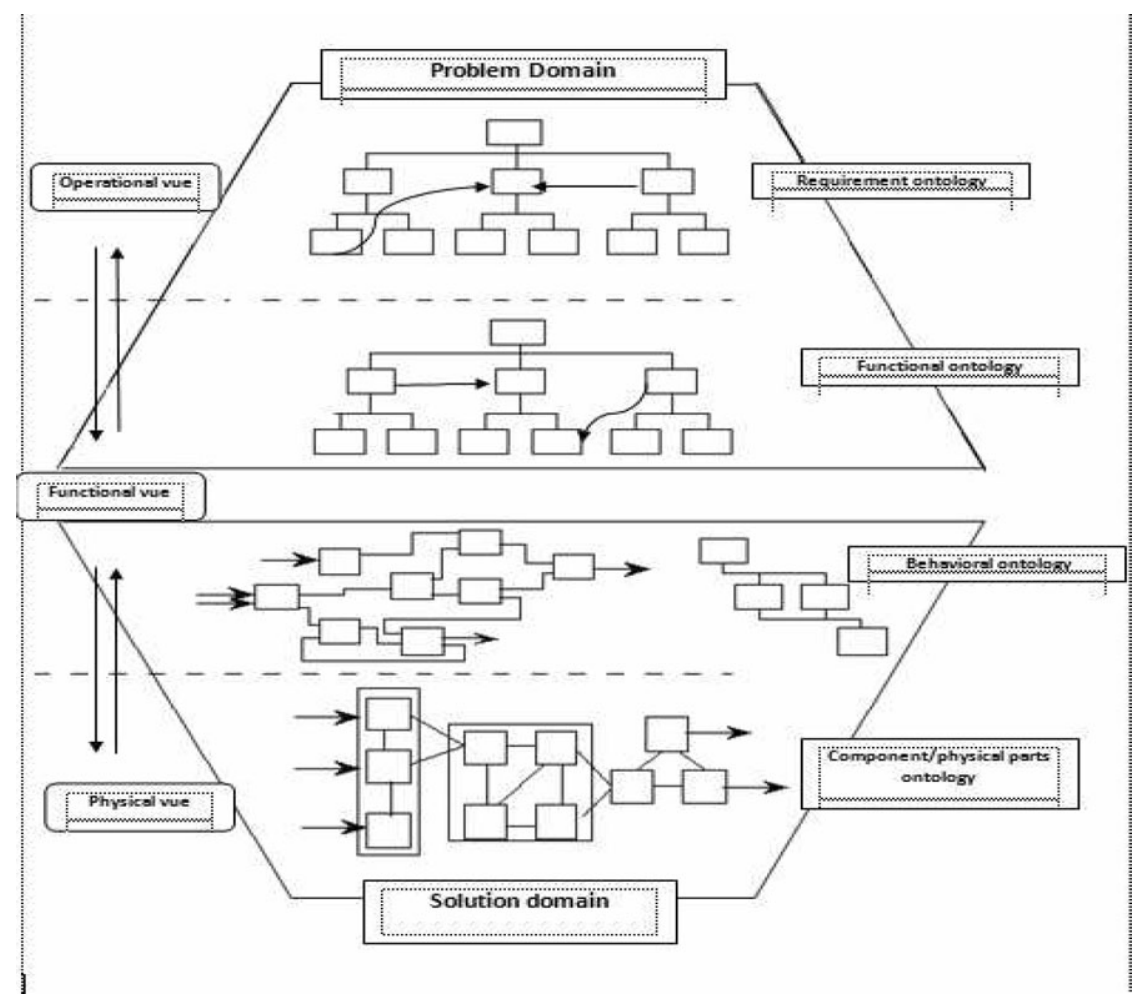

Fig 3: Ontologies for system engineering domain facets

-Process facet: contains concepts and relations that formally describe engineering activities, tasks, actors, and design rationales concepts (intentions, alternatives, argumentations and justification for engineering decisions). Both the process and the product facets act as a formal structure for the SE-Project Asset. The domain facet provides semantic domain values for characterizing this structure. Figure 4, illustrates the relationships and the complementarily of our three modeling facets for comprehensively representing SE-Project Assets.

\subsection{Multi-layered Ontologies for SE Knowledge Modeling}

For the sake of generality of our modeling framework, we have proposed the higher-level concepts for modeling SE-Project Asset. The concepts presented in the above section must be specialized and refined in order to provide operational 
knowledge model for system engineering projects.

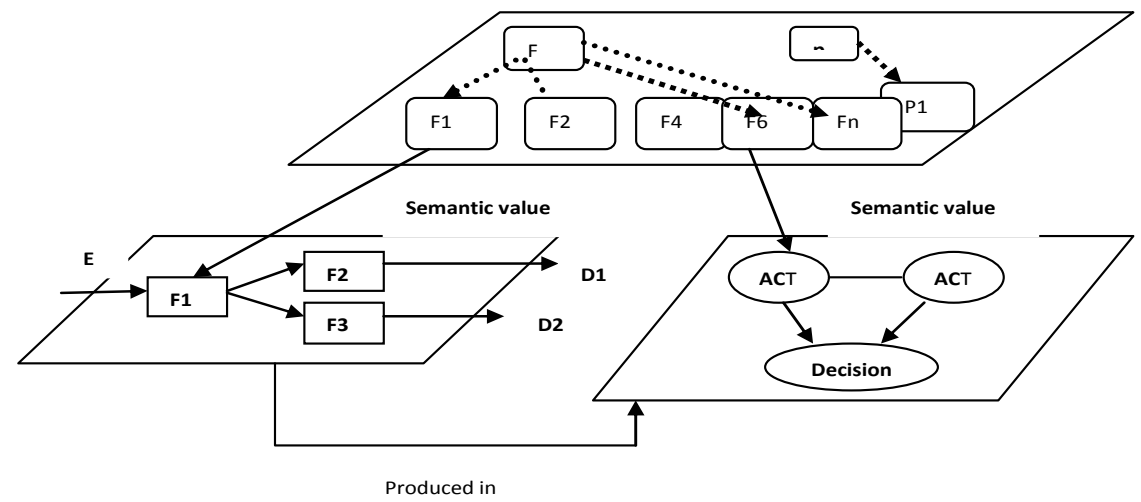

Fig 4: SE general ontology: domain, product and process facet

More precisely, the proposed SE General ontology must be refined in function of the engineering domain (aeronautics, information system, automobile etc.) and in function of the system engineering organizational context.

We propose an ontological framework organized into four semantic layers: layers subdivide the ontology into several levels of abstraction, thus separating general knowledge from knowledge about particular domains, organizations and projects. Basically, knowledge in a certain layer is described in terms of the concepts in the lower layer.

Figure 5 shows a hierarchy of ontologies built on top of SE general ontology.

The first layer aims to describe super-concepts that are the same across all domains, it corresponds to the SE General ontology. The domain layer defines specializing concepts and semantic relations for a system engineering domain such as aeronautics. It integrates for examples domain theories and typical domain concepts that are shared in an engineering community. The application layer, presents specialized concepts used by specific system engineering organization, this is the most specialized level for knowledge characterization and acts as a systematized representation for annotating engineering knowledge projects. The fourth layer corresponds to semantic annotation on SE project assets defined using conceptual vocabulary from the application layer. In this way, all SE project assets are captured as formal knowledge models, by instantiating these ontological concepts. 


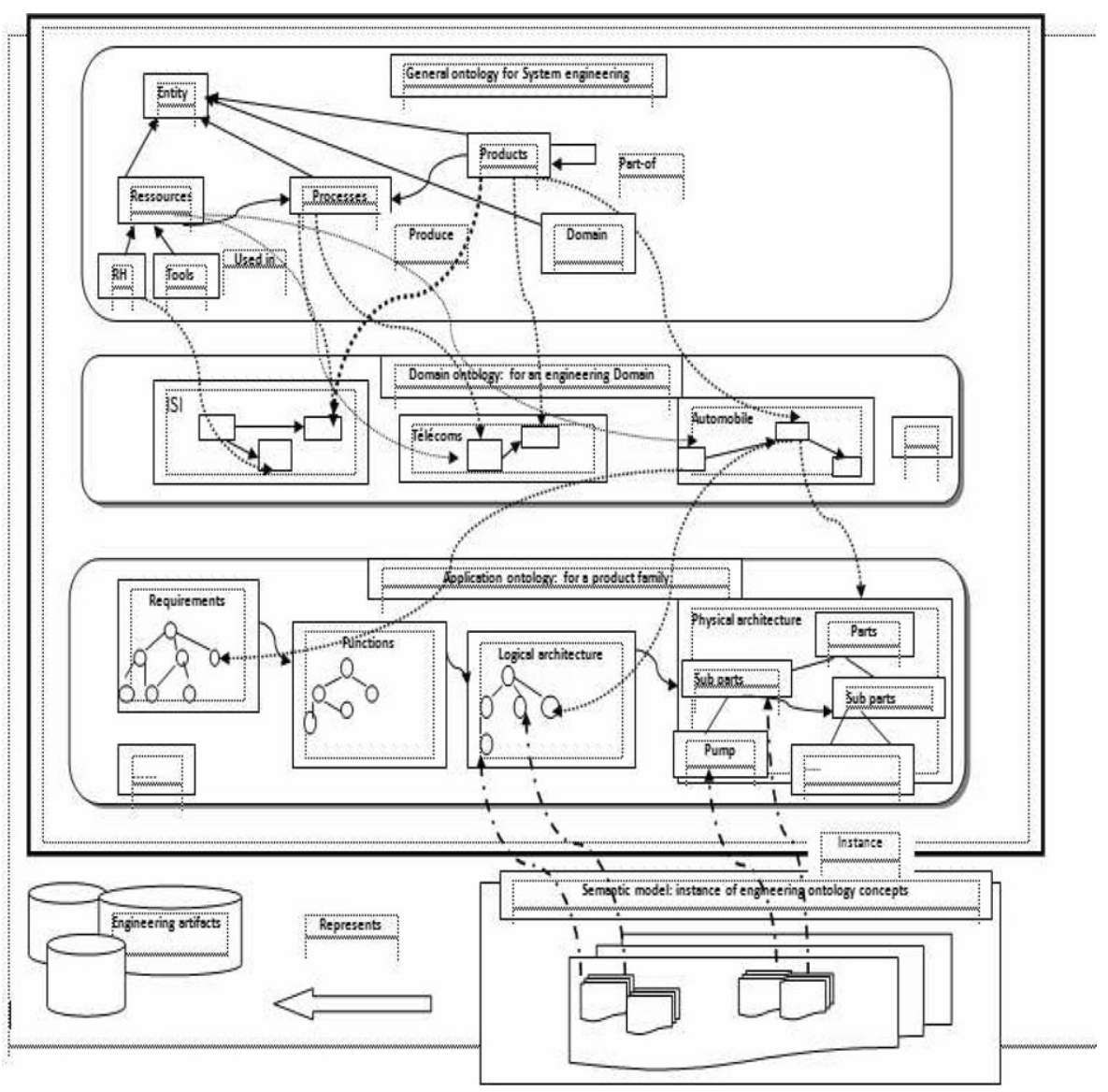

Fig 5: Ontology layers for SE projects

\section{Related work}

Most of the existing SE tools still lack essential aspects needed for supporting knowledge capitalization and reuse during projects processes. To our knowledge, there is no generic framework for knowledge management in SE domain.

In design engineering domain, [16] have integrated concepts of artificial intelligence into commercial PDM systems. The software is based on a dynamic and flexible workflow model, as opposed to the deterministic workflows seen in most commercial PDM applications. [17] Describes an integration of a PDM system with ontological methods and tools. 
The Protégé ontology editor is combined with a commercial PDM system to provide knowledge management capabilities for the conceptual design stage. [18] Have designed ontology for the representation of product knowledge. A Core Ontology defines the basic structure to describe products from a functional view. An ontological architecture for knowledge management that resembles to our proposed framework has been proposed by Sebastian C. Brandt [19] and illustrated in the context of chemical engineering processes. Our knowledge modeling approach, in a way, tries to combine and to extend the ideas underlying the discussed related works into a coherent framework and to tailor them towards the specific system engineering domain and application.

\section{CONCLUSION}

System engineering processes implies the management of information and knowledge and could be considered as a knowledge production process. We have proposed a knowledge modeling framework based on ontologies for capturing in a semantic level the informal engineering artifacts in SE projects. A principal strand of future research is the application of this modeling framework in the context of an engineering organization to trigger further improvement. We plan also to use the same framework for capturing "best practices" knowledge. The problem of providing a knowledge management interface integrated to existing system engineering support tools is also under investigation.

\section{References}

1. J. Meinadier. Le métier d'intégration de systèmes. Hermes Science Publications, décembre 2002.

2. Awad, E. M., \& Ghaziri, H. M. Knowledge management. Prentice Hall. 2003.

3. McMahon, C., Lowe, A., \& Culley, S. J. (2004). Knowledge management in engineering design, personalisation and codification. Journal of Engineering. Design, 15(4), 307-325.

4. Chan-Hong park, M. Kang, J. Ahn, A knowledge representation framework in design repositories. Solid state phenomena Vol 120 (2007), pp. 235-240.

5. Riichiro Mizoguchi, Yoshinobu Kitamura. "Foundation of Knowledge Systematization: Role of Ontological Engineering". The Institute of Scientific and Industrial Research, Osaka University, 8-1, Mihogaoka, Ibaraki, Osaka, 567-0047, Japan.

6. YOSHINOBU KITAMURA. "Roles Of Ontologies Of Engineering Artifacts For Design Knowledge Modeling". In Proc. of the 5th International Seminar and Workshop Engineering Design in Integrated Product Development. (EDIProD 2006), 21-23 September 2006, Gronów, Poland, pp. 59-69, 2006C.

7. A. T. Bahill and B. Gissing, Re-evaluating systems engineering concepts using systems thinking, IEEE Transaction on Systems, Man and Cybernetics, Part C: Applications and Reviews, 28 (4), 516-527, 1998. 
8. Michalis Miatidis, Matthias Jarke. "Toward Improvement-Oriented Reuse of Experience in Engineering Design Processes”. R. Khosla et al. (Eds.): KES 2005, LNAI 3684, pp. 228234, 2005.

9. Robillard, P.N., The role of knolwedge in software development. Communications of the ACM, 1991. 42(1): p. 87-92.

10. Ali-Babar, M., I. Gorton, and B. Kitchenham, A Framework for Supporting Architecture Knowledge and Rationale Management, in Rationale Management in Software Engineering, A. H. Dutoit, et al., Editors. 2005,

11. Bosch, J. Software Architecture: The Next Step. European Workshop on Software Architecture. 2004.

12. Yann pollet. Intégration de systèmes à logiciel prépondérant Groupe « Modélisation des systems complexes »22/10/2007, Conservatoire National des Arts et Métiers Chaire d'intégration des systemes.

13. Gruber, T. R., What is an Ontology?, http://wwwksl stanford.edu/kst/what-is-anontology.html

14. Genesereth, M. and Nilsson, N. Foundation of Artificial Intelligence, 1987.

15. de Kleer, J., Brown, J. S.: A Qualitative Physics Based on Confluences, Artificial Intelligence, Vol.24, 1984, pp.7-83.

16. Kim, Y., Kang, S., Lee, S., \& Yoo, S. (2001). A distributed, open, intelligent product data management system. International Journal of Computer Integrated Manufacturing, 14, 224235.

17. Gao, J. X., Aziz, F. L., Maropoulos, P. G., \& Cheung, W. M. (2003). Application of product data management technologies for enterprise integration. International Journal of Computer Integrated Manufacturing, 16(7-8), 491-500.

18. Kopena, J., \& Regli, W. C. (2003). Functional modeling of engineering designs for the semantic web. IEEE Data Engineering Bulletin, 26(4), 55-61.

19. Sebastian C. Brandt, Jan Morbach, Michalis Miatidis, Manfred Theißen, Matthias Jarke, Wolfgang Marquardt. An ontology-based approach to knowledge management in design processes. Computers and Chemical Engineering (2007). 